TecnoLógicas

ISSN-p 0123-7799

ISSN-e 2256-5337

Vol. 21, No. 43, pp. 53-69

Sep-dic de 2018

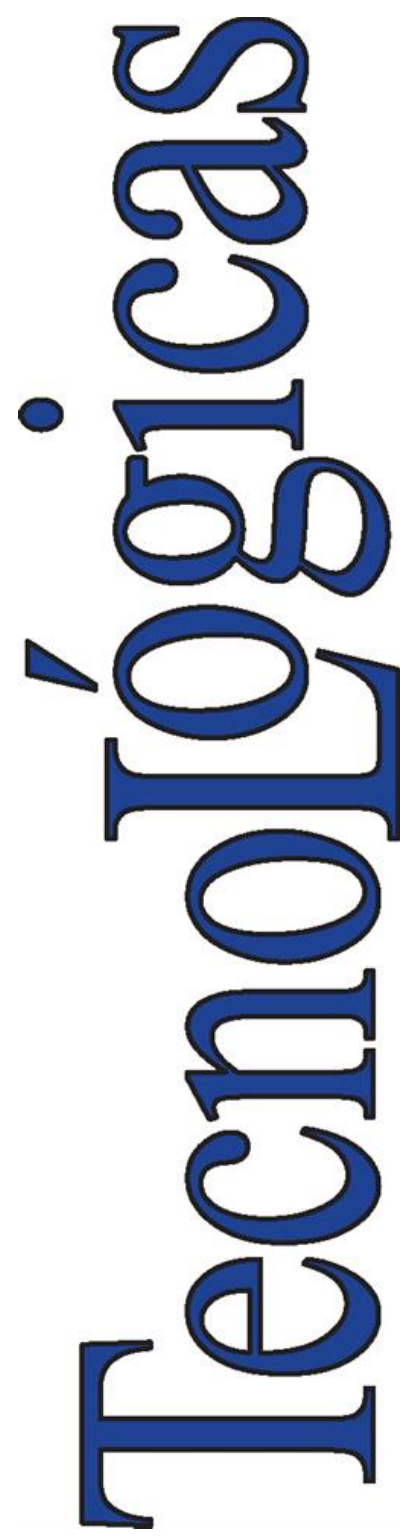

(C) Instituto Tecnológico Metropolitano

Este trabajo está licenciado bajo una Licencia Internacional Creative Commons CC BY-NC-SA

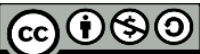

Artículo de Investigación/Research Article

\section{Representaciones tiempo-frecuencia basadas en sensores inerciales para caracterizar la marcha en la enfermedad de Parkinson}

\section{Time-frequency representations from inertial sensors to characterize the gait in Parkinson's disease}

\author{
Marlon E. Bedoya-Vargas ${ }^{1}$, Juan C. Vásquez-Correa², y \\ Juan R. Orozco-Arroyave ${ }^{3}$
}

Recibido: 22 de noviembre de 2017

Aceptado: 10 de mayo de 2018

Cómo citar / How to cite

M. E. Bedoya-Vargas, J. C. Vásquez-Correa, y J. R. Orozco-Arroyave, Representaciones tiempo-frecuencia basadas en sensores inerciales para caracterizar la marcha en la enfermedad de Parkinson. TecnoLógicas, vol. 21 , no. 43 , pp. $53-69,2018$.

1 Ingeniero Electrónico, Grupo de investigación en Telecomunicaciones aplicadas (GITA), Facultad de Ingeniería, Universidad de Antioquia, Medellín-Colombia, marlon.bedoya@udea.edu.co

2 MSc. en Ingeniería de Telecomunicaciones, Grupo de investigación en Telecomunicaciones aplicadas (GITA), Facultad de Ingeniería, Universidad de Antioquia, Medellín-Colombia, Laboratorio de reconocimiento de patrones (LME), Universidad de Erlangen, Erlangen-Alemania, jcamilo.vasquez@udea.edu.co

$3 \mathrm{PhD}$ en Ciencias de la Computación, Grupo de investigación en Telecomunicaciones aplicadas (GITA), Facultad de Ingeniería, Universidad de Antioquia, Medellín-Colombia, Laboratorio de reconocimiento de patrones (LME), Universidad de Erlangen, Erlangen-Alemania, 


\section{Resumen}

La Enfermedad de Parkinson (EP) es un desorden neurodegenerativo del sistema nervioso central, cuyas características principales incluyen entre otras la rigidez, bradicinesia y pérdida de los reflejos posturales. El diagnóstico de la EP está basado en análisis de la historia clínica y evaluaciones físicas realizadas a los pacientes. El monitoreo del estado neurológico de los pacientes está basado en valoraciones subjetivas que realizan los neurólogos. El análisis de la marcha usando sensores inerciales aparece como un instrumento sencillo y útil para ayudar en el proceso de diagnóstico y monitoreo de los pacientes con EP. En este artículo usamos el sistema eGaIT, el cual captura señales de acelerómetro y giróscopo del proceso de marcha para evaluar las habilidades motoras de los pacientes. Las transformadas de Fourier y Wavelet son utilizadas para extraer medidas basadas en energía y entropía en el dominio de Tiempo-Frecuencia. Las características extraídas son utilizadas para discriminar entre pacientes con EP y personas sanas. De acuerdo con los resultados, es posible clasificar estos dos grupos con una precisión de hasta el $94 \%$.

\section{Palabras clave}

Enfermedad de Parkinson, sensores inerciales, representación tiempo-frecuencia, Transformada Wavelet, análisis de marcha, clasificación supervisada.

\section{Abstract}

Parkinson's Disease (PD) is a neurodegenerative disorder of the central nervous system whose main symptoms include rigidity, bradykinesia, and loss of postural reflexes. PD diagnosis is based on an analysis of the medical record and physical examinations of the patient. Besides, the neurological state of patients is monitored with subjective evaluations by neurologists. Gait analysis using inertial sensors was introduced as a simple and useful tool that supports the diagnosis and monitoring of PD patients. This work used the eGaIT system to capture the signals of the accelerometer and the gyroscope of the gait in order to evaluate the motor skills of patients. Fourier and wavelet transform were used to extract measurements based on energy and entropy in the time-frequency domain. The extracted characteristics were used to recognize differences between PD patients and healthy individuals. The results enabled to classify said groups with an accuracy of up to $94 \%$.

\section{Keywords}

Parkinson's Disease, inertial sensors, time-frequency representation, wavelet transform, gait analysis, supervised classification. 


\section{INTRODUCCIÓN}

La Enfermedad de Parkinson (EP) es un desorden neurodegenerativo del sistema nervioso central, caracterizado por la pérdida de neuronas dopaminérgicas [1]. Dentro de sus síntomas, se encuentra de manera más evidente la presencia de temblor de reposo, rigidez, bradicinesia y pérdida de los reflejos posturales. Otros síntomas observados y documentados en la literatura incluyen micrografía en la escritura, así como monotonicidad y disartria en la voz [2]. La herramienta utilizada comúnmente para evaluar el estado neurológico de la EP es la escala MDS-UPDRS (por las siglas en inglés de Movement Disorder Society - Unified Parkinson's Disease Rating Scale) [3,4], la cual está distribuida en 4 secciones: parte I: mental, conductual y de ánimo; parte II: actividades de la vida diaria; parte III: evaluación motora; y parte IV: complicaciones motoras. La parte 3 de la escala contiene 33 ítems y cada uno se evalúa entre 0 (completamente sano) y 4 (totalmente afectado, sin capacidad de movimiento), formando una escala completa entre 0 y 132. Cada uno de los ítems evalúa tareas como el golpeteo de los dedos en las manos, golpeteo con los dedos de los pies, agilidad de las piernas, postura, levantarse de la silla, congelamiento de la marcha, persistencia de temblor en reposo, entre otras [4].

$\mathrm{El}$ estudio específico del movimiento de las extremidades inferiores ha resultado ser de interés general para la comunidad científica debido a su sensibilidad a la hora de efectuar análisis motor en pacientes con EP [5]. Este tipo de estudios están basados en la captura y procesamiento de señales generadas por sensores inerciales compuestos por acelerómetros y giróscopos adheridos al pie de los pacientes. Dichas señales son capturadas mientras las personas realizan tareas previamente diseñadas por neurólogos expertos.
Diferentes investigaciones demuestran que es posible extraer patrones característicos del movimiento de las extremidades inferiores tales que permitan relacionar las medidas obtenidas con el estado neurológico de los pacientes.

En [6] se capturan señales de marcha de pacientes con EP y personas sanas usando sensores inerciales adheridos al zapato del paciente, y se usa la transformada de Fourier para extraer características basadas en la energía relativa en distintas bandas de frecuencia, y medidas estadísticas como media, varianza, máximo de autocorrelación, entre otras. Los autores implementaron un clasificador basado en máquinas de soporte vectorial (SVM, por sus siglas en inglés) y AdaBoost, y reportan aciertos de hasta el $91 \%$ con las características calculadas en la marcha. En [7], los autores realizan un análisis de marcha usando sensores inerciales en 10 pacientes con EP y 10 personas sanas. Se calculan características espacio-temporales, como la velocidad y longitud de cada paso, el ángulo de rotación, entre otras. Los autores analizan la diferencia entre las características de un grupo de control, pacientes estado $\mathrm{ON}, \mathrm{y}$ pacientes en estado OFF, y encuentran diferencias significativas usando el test de Wilcoxon de rango para algunas de las características evaluadas en tareas de marcha de 20 metros ida y vuelta. En [8] se extraen características en los dominios de tiempo, frecuencia y tiempo-frecuencia. Los autores usan los test estadísticos KruskalWallis y Mann-Whitney para comparar un grupo de pacientes con EP, un grupo de pacientes con alguna patología neurodegenerativa diferente y un grupo de sujetos sanos. Los autores usan la transformada wavelet y calculan características de entropía, centroides espectrales y energía por banda de frecuencia. Los resultados indican que hay diferencias significativas cuando se considera la entropía $(\mathrm{p}<<0.001)$, la concentración de energía por banda 
wavelet $(\mathrm{p}<<0.02)$, y $\quad$ los centroides espectrales $(p<<0.04)$, concluyendo que dichas medidas aportan información significativa para discriminar entre pacientes y personas sanas. En [9] se hace uso de acelerómetros y giróscopos para calcular medidas espacio-temporales, como la longitud, velocidad y el tiempo de cada paso, el tiempo de fase de postura, la compensación del pie, y variación del ángulo del golpeteo del talón para determinar diferencias grupales e individuales en los parámetros de la marcha entre una población de pacientes con EP y personas sanas. Los autores reportan diferencias de hasta el $24 \%$ en el ángulo del pie cuando es levantado para hacer el golpeteo de talón. En [10] se extraen características de voz, escritura y marcha para clasificar entre pacientes y controles, y predecir el estado neurológico de los pacientes. En el caso de la marcha, se calcularon características biomecánicas, incluyendo la longitud del paso, la velocidad de la marcha, la cadencia, el ángulo de rotación y el número de pasos. Los autores reportan un coeficiente de correlación de Spearman de hasta 0.72 en la predicción del estado neurológico con base en la escala MDS-UPDRS-III.

En este estudio se analiza la marcha de pacientes con EP usando sensores inerciales ubicados en los zapatos de los participantes. A partir de las señales capturadas, se realiza un análisis tiempofrecuencia y se calculan características basadas en energía y entropía en dos representaciones: la transformada de Fourier de tiempo corto (STFT, por las siglas en Inglés de Short Time Fourier Transform) y la transformada wavelet continua (CWT, por las siglas en inglés de Continuous Wavelet Transform). Adicionalmente, se calculan características de perturbación en el tiempo, como el shimmer, el jitter y la distorsión armónica total. Por último, se usa una SVM lineal de margen suave para clasificar entre pacientes con EP y dos grupos de personas sanas con dos rangos de edades diferentes. De acuerdo con los resultados, es posible clasificar entre pacientes y personas sanas con una tasa de aciertos de hasta el $94 \%$.

Además, se evalúa que las medidas analizadas en este estudio proveen información relevante para caracterizar el efecto de la EP en la marcha

\section{MÉTODOS}

\subsection{Análisis de perturbación temporal}

Las medidas de perturbación buscan medir la variabilidad de la amplitud y del periodo de la señal a lo largo del tiempo. En este estudio se extraen medidas de jitter y de Shimmer para analizar las perturbaciones en frecuencia y en amplitud de la señal a lo largo del tiempo [10]. Adicionalmente, se calcula la distorsión armónica total (THD, por las siglas en Inglés de Total Harmonic Distortion) y la localización de los 3 máximos de amplitud en el tiempo. La Tabla 1 incluye las expresiones utilizadas para el cálculo de las características temporales. La Fig. 1 muestra un tramo de una señal de aceleración normalizada en el dominio del tiempo para una persona sana (izquierda) y para un paciente con MDS-UPDRS=82 (derecha). A partir de las señales mostradas en la Fig. 1, se realiza un enventanado de dos segundos de duración para el cálculo del Shimmer y del jitter. Esta duración de ventana es fijada en dos segundos, con el fin de garantizar que existan al menos tres quasiperiodos de la señal. Estas características se calculan como medidas totales para toda la señal en el tiempo y también se calculan de manera dinámica en grupos de tres ventanas consecutivas, obteniendo así el comportamiento de dichas características a lo largo del tiempo. 
Representaciones tiempo-frecuencia basadas en sensores inerciales para caracterizar la marcha en la enfermedad de Parkinson

Tabla 1. Expresiones utilizadas para el cálculo de las características en el dominio temporal. $\boldsymbol{A}_{\boldsymbol{i}}:$ Amplitudes. $\boldsymbol{T}_{\boldsymbol{i}}$ : Períodos. $\boldsymbol{I}_{\boldsymbol{i}}$ : Armónicos de frecuencia. Fuente: autores.

\begin{tabular}{|c|c|c|}
\hline Medida & Expresión matemática & Descripción \\
\hline Shimmer absoluto (dB) & $\operatorname{Shimmer}(d B)=\frac{1}{N-1} \sum_{i=0}^{N-1}\left|20 \log \frac{A_{i+1}}{A_{i}}\right|$ & $\begin{array}{l}\text { Expresa la variabilidad pico a pico } \\
\text { de la amplitud en decibeles a lo } \\
\text { largo de la señal. }\end{array}$ \\
\hline Shimmer relativo & $\begin{array}{l}\text { Shimmer(relativo) } \\
=\frac{\frac{\mathbf{1}}{N-\mathbf{1}} \sum_{i=\mathbf{0}}^{N-1}\left|A_{i}-A_{i-1}\right|}{\frac{\mathbf{1}}{N} \sum_{i=0}^{N} A_{i}}\end{array}$ & $\begin{array}{l}\text { Está dado por el shimmer absoluto } \\
\text { dividido entre el promedio de las } \\
\text { amplitudes de la señal medida en } \\
\text { todo el tiempo. }\end{array}$ \\
\hline Jitter absoluto & Jitter(absoluto) $=\frac{1}{N-1} \sum_{i=0}^{N-1}\left|T_{i}-T_{i+1}\right|$ & $\begin{array}{l}\text { Es la variación del periodo } \\
\text { fundamental de la señal ciclo a } \\
\text { ciclo. }\end{array}$ \\
\hline Jitter relativo & $\operatorname{Jitter}($ relativo $)=\frac{\frac{1}{N-1} \sum_{i=0}^{N-1}\left|T_{i}-T_{i+1}\right|}{\frac{1}{N} \sum_{i=0}^{N} T_{i}}$ & $\begin{array}{l}\text { El jitter relativo es el jitter } \\
\text { absoluto dividido por el promedio } \\
\text { de los periodos. }\end{array}$ \\
\hline Distorsión armónica total & $\mathbf{T H D}=\frac{\sqrt{\sum_{n=2}^{\infty} I_{n}^{2}}}{I_{1}}$ & $\begin{array}{l}\text { Potencia de todos los armónicos } \\
\text { entre la potencia de la frecuencia } \\
\text { fundamental. }\end{array}$ \\
\hline
\end{tabular}
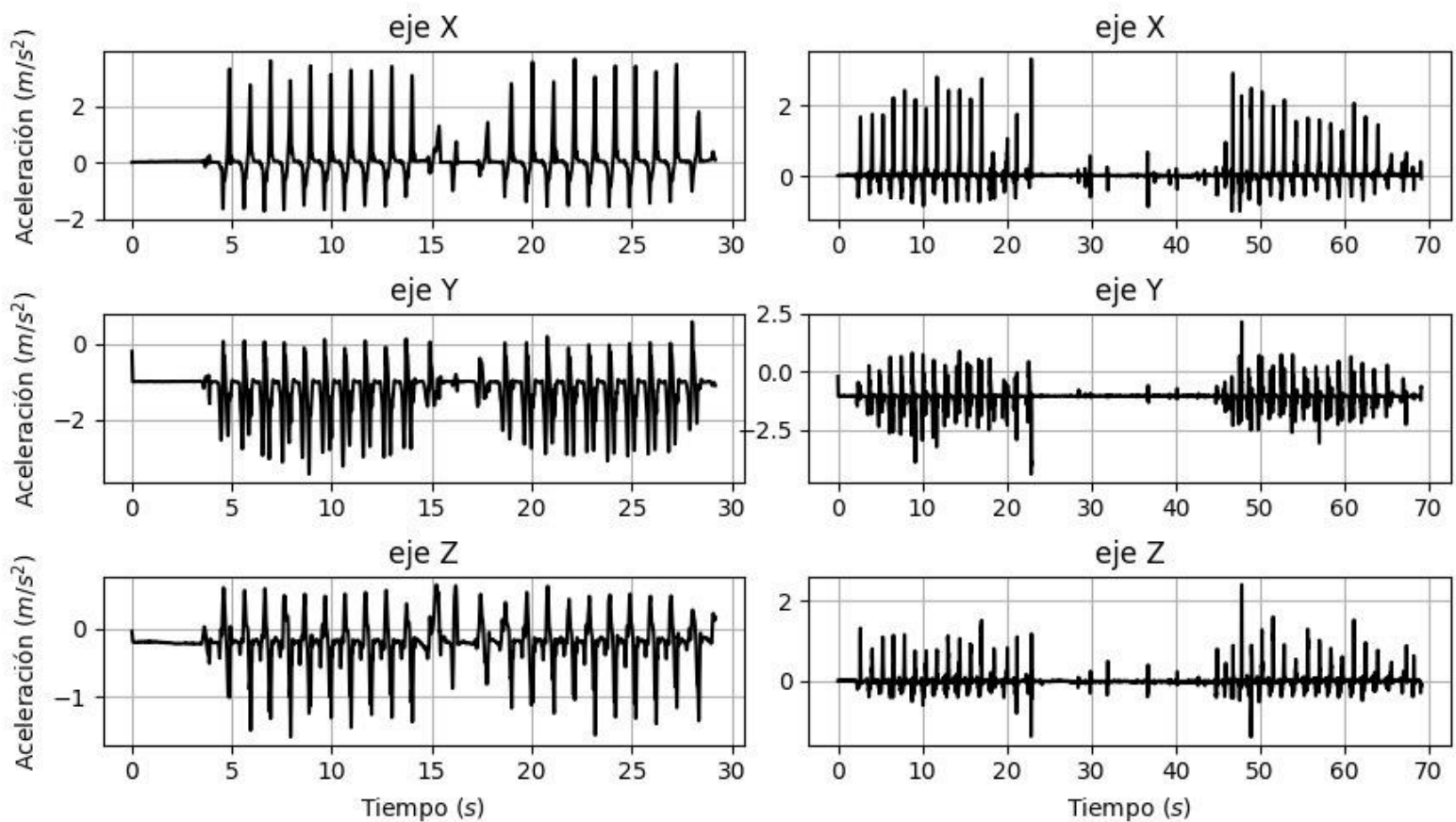

Fig. 1. Señales de aceleración en el pie izquierdo para persona sana, hombre de 64 años (izquierda) y paciente con EP (derecha), hombre de 62 años y con MDS-UPDRS-III de 82. Fuente: autores. 


\subsection{Transformada de Fourier en tiempo corto}

La STFT es una versión corta y comúnmente enventanada de la transformada de Fourier para señales discretas, en la cual se calcula la transformada de Fourier de las muestras temporales que aparecen en intervalos cortos de tiempo de la señal original.

Típicamente se aplica una ventana sobre cada intervalo de señal, previo al cálculo de la transformada de Fourier. El objetivo principal de dicho enventanado es evitar cambios bruscos tales que puedan generar discontinuidades en los intervalos cortos de tiempo definidos para analizar la señal original. De esta forma, se obtienen espectros consecutivos de zonas reducidas de la secuencia de entrada, cada una de ellas de longitud igual a la de la ventana y centradas en el tiempo [11]. De esta manera, si se quiere realizar un análisis de la dinámica de la señal en un intervalo de tiempo particular, entonces se toma una pequeña porción de la señal centrada alrededor de dicho intervalo y se calcula el espectro en la vecindad [12]. Dicha transformada está dada por (1), donde $\boldsymbol{\omega}(\boldsymbol{t}-\boldsymbol{m})$ es la ventana de análisis desplazada un tiempo $\boldsymbol{m}$

$\operatorname{STFT}\{X\{t\}\}=\int_{-\infty}^{\infty} x(t) \omega(t-m) e^{-j \omega t} d t$

Para la visualización o para etapas posteriores de procesamiento, se usa el espectrograma, que es el cuadrado de la magnitud de la STFT, tal como se expresa en (2).

$$
T_{S P E C T}=|\operatorname{STFT}\{X(t)\}|^{2}
$$

La Fig. 2 muestra espectrogramas obtenidos para una persona sana (izquierda), y para un paciente con MDSUPDRS=82. Se puede apreciar que el paciente tiene mayor concentración de energía en la zona de alta frecuencia, respecto a la persona sana. Note además que en la persona sana es posible distinguir instantes de tiempo donde la persona realiza pausas, lo que no es posible apreciar en el paciente. Este comportamiento puede indicar la dificultad de los pacientes al momento de iniciar, finalizar el movimiento, lo que ha sido observado en trabajos previos con señales de voz [13].

\subsection{Transformada Wavelet Continua (CWT)}

Una wavelet es una forma de onda de duración limitada que tiene un valor promedio cero. El análisis wavelet, análogo al análisis de Fourier, descompone la señal en versiones escaladas y desplazadas de la wavelet original o madre $\boldsymbol{\psi}(\boldsymbol{t})$ que representa el mismo papel que $\boldsymbol{e}^{-j \omega t}$ en la definición de la transformada de Fourier.

La CWT se define como la suma en el tiempo de la señal wavelet Madre escalada y desplazada [14]. La expresión general para la CWT se define de acuerdo con (3), donde a es el factor de escala y b es el factor de traslación.

$T_{x}(a, b)=<x, \psi_{a, b}>=\frac{1}{\sqrt{a}} \int_{-\infty}^{\infty} x(t) \psi\left(\frac{t-b}{a}\right)$

Las wavelets $\boldsymbol{\psi}_{\boldsymbol{a}, \boldsymbol{b}}$ generadas de la misma función wavelet madre $\boldsymbol{\psi}(\boldsymbol{t})$ tienen diferente escala $\boldsymbol{a}$ y ubicación $\boldsymbol{b}$, pero tienen todas la misma forma. Cambiando el valor de $\boldsymbol{a}$ se cubren rangos diferentes de frecuencias. Valores grandes de $\boldsymbol{a}$ corresponden a frecuencias bajas, o una escala grande de $\boldsymbol{\psi}_{\boldsymbol{a}, \boldsymbol{b}}(\boldsymbol{t})$. Valores pequeños de $\boldsymbol{a}$ corresponden a frecuencias altas, o una escala muy pequeña de $\boldsymbol{\psi}_{\boldsymbol{a}, \boldsymbol{b}}(\boldsymbol{t})$ [15].

Este tipo de transformada permite analizar señales transitorias y no estacionarias con mejor resolución que la STFT. Lo anterior dado que con la STFT se tiene una resolución fija de tiempo y frecuencia, mientras que la CWT permite 
adaptar el ancho de la ventana en función de las frecuencias y el tiempo [16].

La Fig. 3 muestra la CWT para la misma persona sana y paciente que la Fig. 2. Se puede apreciar el mismo efecto observado en la STFT. En el paciente no es posible distinguir el momento de inicio y fin del movimiento, lo que sí se puede apreciar en la persona sana (izquierda).

\subsection{Características extraídas en el dominio Tiempo-Frecuencia}

En este estudio, se calculan espectrogramas con un tamaño de ventana de 0.5 segundos y una resolución de 512 puntos de 0 a $50 \mathrm{~Hz}$. Luego, se divide en 20 bandas de $2.5 \mathrm{~Hz}$ cada una con la finalidad de obtener medidas relativas por banda.
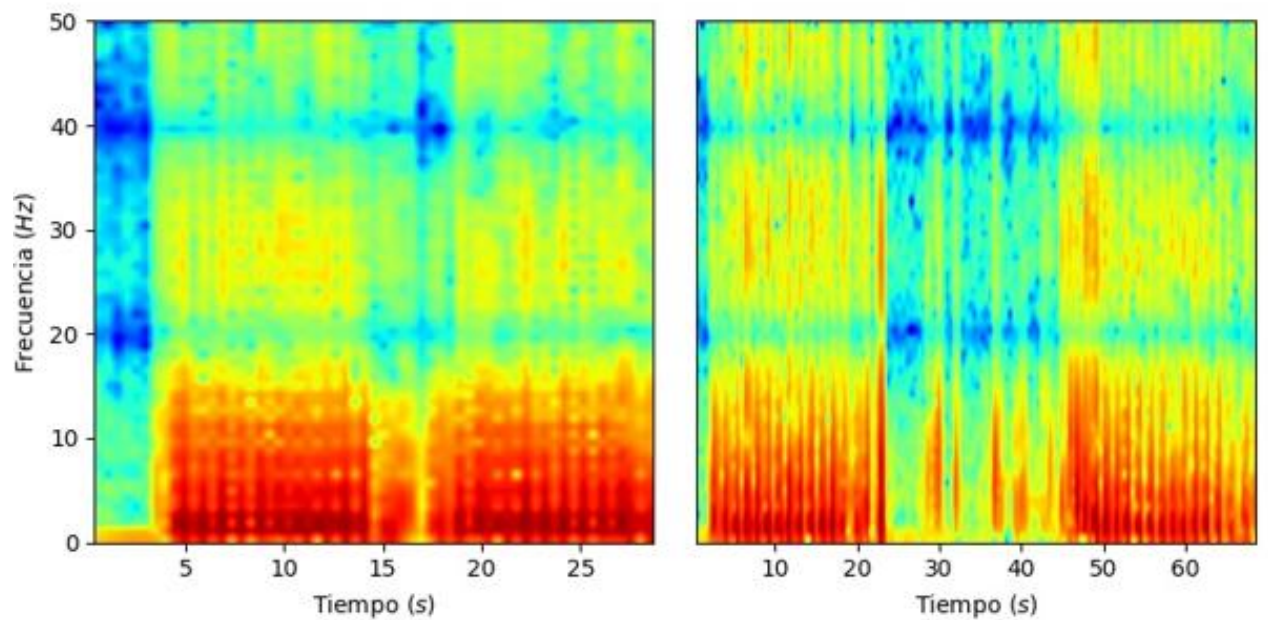

Fig. 2. Representaciones y resoluciones las transformada STFT para Control sano, hombre de 64 años (izquierda), y paciente con EP, hombre, 62 años y MDS-UPDRS-III de 82 (derecha). Fuente: autores.
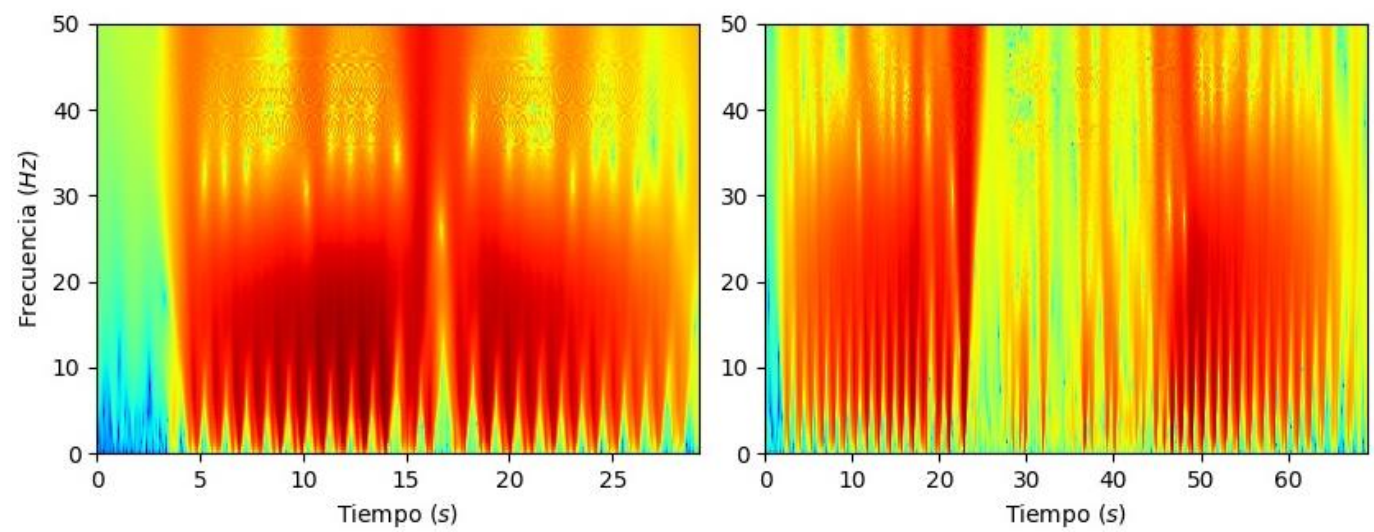
Representaciones tiempo-frecuencia basadas en sensores inerciales para caracterizar la marcha en la enfermedad de Parkinson

Fig. 3. Representaciones y resoluciones las transformada CWT para Control sano, hombre de 64 años (izquierda), y Paciente con EP, hombre, 62 años y MDS-UPDRS-III de 82 (derecha). Fuente: Autores.

Sobre cada representación tiempofrecuencia obtenida de la CWT y la STFT, y sobre cada una de las bandas de frecuencia establecidas, se calculan cuatro grupos de características que pueden se observan en la Tabla 2. Las características incluyen la energía absoluta y relativa en cada banda de frecuencia, la entropía espectral, y tres centroides espectrales. Cada característica es calculada sobre cada uno de los seis ejes de los sensores inerciales utilizados (acelerómetro de 3 ejes y giróscopo de 3 ejes).

\subsection{Clasificación automática}

En este estudio se ha utilizado una SVM de margen blando como algoritmo de clasificación entre los pacientes y los dos grupos de controle establecidos.

El objetivo de una SVM es discriminar las muestras de cada clase establecida usando un hiperplano separador que maximiza el margen de separación ente las clases. La función de decisión de la SVM se expresa de acuerdo con (4). La SVM de margen suave es conocida por permitir algunos errores en el proceso de optimización para encontrar el hiperplano de separación. El término $\xi_{i}$ es una variable de holgura que penaliza la cantidad de errores permitidos en el proceso de optimización. $y_{i} \in\{-1,+1\}$ son las etiquetas de cada muestra, $\phi\left(\boldsymbol{x}_{\boldsymbol{i}}\right)$ es una función kernel que transforma el espacio de características $\boldsymbol{x}$ en un espacio de mayor dimensión, donde una separación lineal pueda ser realizada. El vector de pesos $\boldsymbol{w}$ y el término independiente $b$ son los parámetros que definen el hiperplano de separación.

$$
y_{i}\left(\boldsymbol{w}^{T} \phi\left(\boldsymbol{x}_{\boldsymbol{i}}\right)+b\right) \geq 1-\xi_{i}, i=1,2,3, \ldots, N(4)
$$

El problema de optimización que encuentra el hiperplano está dado por (5), donde el hiperparámetro $C$ compensa los errores $\xi_{i}$ y el ancho del margen. Las muestras $\boldsymbol{x}_{\boldsymbol{i}}$ donde se cumple la condición $y_{i}\left(\boldsymbol{w}^{T} \phi\left(\boldsymbol{x}_{\boldsymbol{i}}\right)+b\right)=1-\xi_{i}$ son llamados vectores de soporte $\boldsymbol{x}_{\boldsymbol{m}}$.

Tabla 2. Expresiones a utilizar en las medidas de energía y entropía. $\boldsymbol{M}$ : Número de bandas de frecuencia. T: Tiempo. $\boldsymbol{k}$ : Banda de energía. $\boldsymbol{X}(\boldsymbol{i}, \boldsymbol{k})$ : Coeficiente de la transformada tiempo-frecuencia. Fuente: autores.

\begin{tabular}{|c|c|c|}
\hline Magnitud calculada & Función & Descripción \\
\hline Energía absoluta & $E(k)=\frac{1}{M T} \sum_{k=1}^{T} \sum_{i=1}^{M}|X(i, k)|^{2}$ & $\begin{array}{l}\text { Mide la energía total contenida en la señal en todo } \\
\text { el rango de frecuencias. }\end{array}$ \\
\hline Energía relativa & $E_{r}(k)=\frac{E(k)}{\frac{1}{N} \sum_{k=1}^{T} \sum_{i=1}^{M}|X(i, k)|^{2}}$ & $\begin{array}{l}\text { Mide la contribución de energía en cada banda } \\
\text { tiempo-frecuencia. }\end{array}$ \\
\hline Entropía espectral & $\begin{array}{r}S(x)=\frac{-1}{T} \sum_{k=1}^{T} \sum_{i=1}^{M} X(i, k) \\
* \log |X(i, k)|\end{array}$ & $\begin{array}{l}\text { Cuantifica la cantidad de información en la señal en } \\
\text { el dominio tiempo-frecuencia. Altos valores } \\
\text { representan mayor cantidad de información. }\end{array}$ \\
\hline $\begin{array}{l}\text { Centroides } \\
\text { espectrales }\end{array}$ & $C E=\frac{\int_{0}^{f_{\max }} f\left|F_{k}(f)\right|^{2}}{\int_{0}^{f_{\max }}\left|F_{k}(f)\right|^{2}}$ & $\begin{array}{l}\text { La frecuencia que divide la distribución espectral de } \\
\text { potencia en partes iguales. Se usa cuartiles al } 25 \% \text {, } \\
50 \% \text { y } 75 \% \text { de la energía total. }\end{array}$ \\
\hline
\end{tabular}


$\underbrace{\operatorname{minimizar}}_{\boldsymbol{w}, b} \frac{1}{2}\|\boldsymbol{w}\|^{2}+C \sum_{i=1}^{N} \xi_{i}$

sujeto a

$y_{i}\left(\boldsymbol{w}^{T} \phi\left(\boldsymbol{x}_{\boldsymbol{i}}\right)+b\right) \geq 1-\xi_{i} \geq 0$

\section{MARCO EXPERIMENTAL}

\subsection{Base de datos}

En este estudio se utilizan 3 bases de datos. La primera está compuesta por un grupo de 30 pacientes con EP (11 hombres y 19 mujeres) con promedio de edad de 61.7 años los hombres, y 66.1 años las mujeres. Todos los pacientes han sido diagnosticados y evaluados por un neurólogo experto, de acuerdo con la escala MDS-UPDRS-III. Cada paciente firmó un consentimiento informado aprobado por el comité de bioética de la Universidad de Antioquia. Adicionalmente, todos los pacientes fueron grabados en estado $\mathrm{ON}$, es decir, bajo el efecto de la medicación.

El segundo grupo de sujetos está formado por personas sanas con edades similares a las de los pacientes (21 hombres con edad promedio de 67.4 años y 18 mujeres con edad promedio de 60.5 años). Ninguno de los controles tiene historia de enfermedades neurodegenerativas, o algún otro desorden de movimiento.

Finalmente, el tercer grupo de sujetos está formado por 37 personas sanas jóvenes, con edades entre los 20 y 42 años, también sin historial de enfermedades neurodegenerativas o desórdenes de movimiento. Las señales de este grupo de personas sanas se adquirieron con el fin de evaluar la influencia de la edad en el problema de clasificación abordado, y en general en los desórdenes de movimiento, que ha sido abordado previamente usado señales de voz [17]. La Tabla 3 contiene información detallada sobre las tres bases de datos utilizadas. Cada participante realiza 3 ejercicios, cuya duración promedio es $35 \pm 21 \mathrm{~s}$, durante los cuales se capturan las señales de aceleración lineal y angular en los ejes $x, y$ y $z$ para cada uno de los pies del participante.

Ejercicio 1 (4x10): Marcha en línea recta ida y vuelta en un trayecto de 10 metros en 2 repeticiones. Longitud total de recorrido 40 metros.

Ejercicio 2 (Tapping pie izquierdo): Consiste en tocar alternativamente con la punta y el talón del pie izquierdo el piso mientras la persona se encuentra sentada en una silla. El pie derecho debe estar en reposo. El tiempo total de cada muestra es de 20 segundos.

Ejercicio 3 (Tapping pie derecho): Análogo al Ejercicio 2, pero con el pie derecho en el ejercicio y el pie izquierdo en reposo.

Estos ejercicios se realizan con base en trabajos relacionados de análisis de marcha de pacientes con EP [6,7]. Además, estos ejercicios hacen parte de los ítems incluidos en la escala MDS-UPDRS-III, asignada por los neurólogos en su evaluación.

Las muestras han sido tomadas con el sistema eGaIT, cuyos acelerómetros tienen un rango de $6 \mathrm{~g}$ (sensibilidad $300 \mathrm{mV} / \mathrm{g}$ ) y los giróscopos tienen un rango de 6500 grados/segundo (sensibilidad $2 \mathrm{mV} /$ grado /segundo), a una frecuencia de muestreo de $102.4 \mathrm{~Hz}$ y con 12 bits de cuantización. La Fig. 4 muestra un esquema del sistema utilizado para la captura de los datos.

\subsection{Clasificación}

Se usa una SVM con kernel lineal, cuyo parámetro de penalización $C \in$ $\left\{10 e^{-3}, \ldots, 10 e^{4}\right\}$ es optimizado realizando una malla de búsqueda, usando una validación cruzada de diez particiones, donde se usaron ocho particiones para hacer el entrenamiento, una para 
Representaciones tiempo-frecuencia basadas en sensores inerciales para caracterizar la marcha en la enfermedad de Parkinson

optimizar el hiperparámetro $C$ de la SVM, $\quad$ y la última partición para hacer la prueba.

Tabla 3. Resumen de base de datos utilizada para el desarrollo del proyecto. p: Promedio. o: Desviación estándar.

\begin{tabular}{lcccccc}
\hline & \multicolumn{2}{c}{ Pacientes } & \multicolumn{2}{c}{ Controles jóvenes } & \multicolumn{2}{c}{ Controles adultos } \\
& hombres & mujeres & hombres & mujeres & hombres & mujeres \\
\hline $\begin{array}{l}\text { Número de } \\
\text { participantes }\end{array}$ & 11 & 19 & 21 & 16 & 21 & 18 \\
Edad $(\boldsymbol{\mu} \pm \boldsymbol{\sigma})$ & $61.7 \pm 6.8$ & $66.1 \pm 8.5$ & $25.3 \pm 4.5$ & $23.1 \pm 3.0$ & $67.4 \pm 12.8$ & $60.5 \pm 8.0$ \\
Rango de edad & $48-83$ & $25-75$ & $21-42$ & $20-32$ & $49-84$ & $50-74$ \\
MDS-UPDRS-III $(\boldsymbol{\mu} \pm \boldsymbol{\sigma})$ & $35.63 \pm 24.1$ & $31.35 \pm 13.7$ & - & - & - & - \\
\hline
\end{tabular}

A.

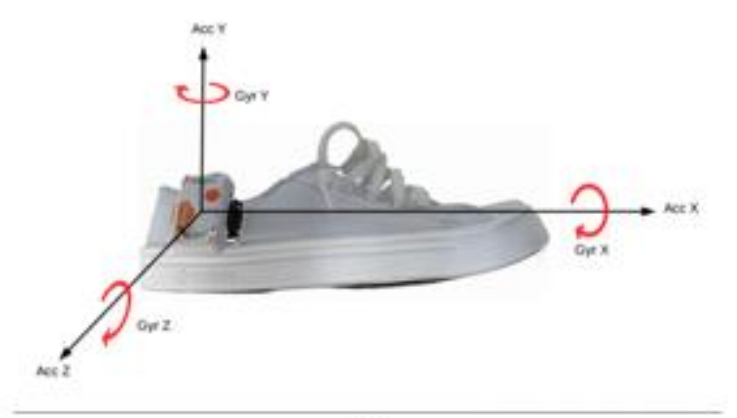

B.

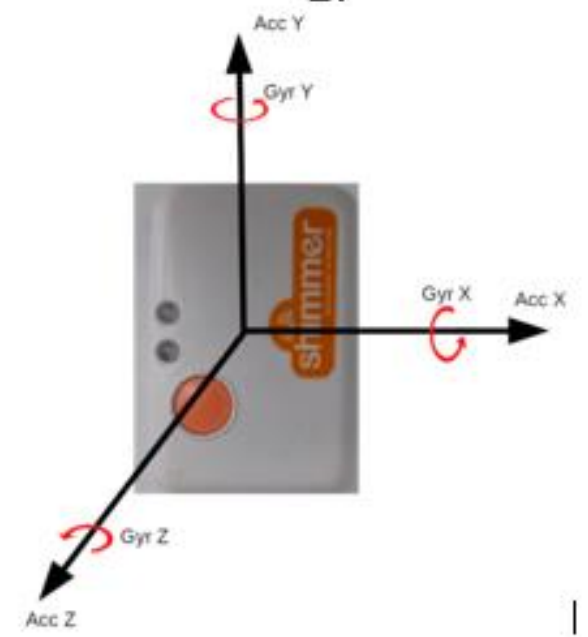

Fig. 4. Sistema utilizado para la captura de los datos. A. Ubicación del sensor en la parte lateral externa del zapato. B. Detalle del sensor utilizado. Fuente: autores

\section{RESULTADOS Y DISCUSIÓN}

\subsection{Clasificación}

Los resultados de la clasificación mostrados en la Tabla 4 describen el porcentaje de acierto, sensibilidad $y$ especificidad que se realiza la clasificación entre pacientes y personas sanas jóvenes, y entre pacientes y personas sanas adultos usando las características tiempo frecuencia a partir de la STFT. Cuando se considera la fusión de todas las características, los mejores resultados en la población de jóvenes se consiguen con el ejercicio $4 \times 10$ (89\%), mientras que en la 
población de adultos se consiguen con el

ejercicio tapping en ambos pies (88\%).

Tabla 4. Resultados totales y parciales de la clasificación en el dominio tiempo frecuencia usando la STFT. FC: Fusión de características $\boldsymbol{E}_{\boldsymbol{a}}$ : Energía absoluta. $\boldsymbol{E}_{\boldsymbol{r}}$ : Energía relativa. $\boldsymbol{S}$ : Entropía espectral. $\boldsymbol{C} \boldsymbol{E}$ : Centroides espectrales. Fuente: autores

\begin{tabular}{|c|c|c|c|c|c|c|}
\hline \multirow[t]{2}{*}{ Características tarea } & \multicolumn{3}{|c|}{$\begin{array}{c}\text { Pacientes Vs } \\
\text { Jóvenes }\end{array}$} & \multicolumn{3}{|c|}{$\begin{array}{c}\text { Pacientes Vs } \\
\text { Adultos }\end{array}$} \\
\hline & Acc & Sen & Spe & Acc & Sen & Spe \\
\hline$F C-4 \mathrm{X} 10$ & 79 & 70 & 86 & 65 & 60 & 69 \\
\hline$F C$ - Tapping & 83 & 77 & 89 & 88 & 80 & 95 \\
\hline$F C-4 \times 10+$ tapping & 76 & 63 & 86 & 73 & 67 & 79 \\
\hline$E_{a}-4 \mathrm{X} 10$ & 89 & 87 & 92 & 73 & 67 & 79 \\
\hline$E_{a}-$ Tapping & 85 & 77 & 92 & 81 & 70 & 90 \\
\hline$E_{a}-4 \times 10+$ tapping & 86 & 80 & 92 & 78 & 67 & 87 \\
\hline$E_{r}-4 \mathrm{X} 10$ & 85 & 63 & 81 & 68 & 47 & 74 \\
\hline$E_{r}$-Tapping & 76 & 60 & 86 & 92 & 70 & 90 \\
\hline$E_{r}-4 \mathrm{x} 10+$ tapping & 83 & 70 & 81 & 84 & 60 & 82 \\
\hline$S-4 \mathrm{X} 10$ & 85 & 83 & 86 & 71 & 63 & 77 \\
\hline$S$ - Tapping & 89 & 87 & 92 & 88 & 77 & 97 \\
\hline$S-4 \times 10+$ tapping & 89 & 80 & 97 & 76 & 67 & 85 \\
\hline$C E-4 \mathrm{X} 10$ & 73 & 77 & 92 & 62 & 63 & 64 \\
\hline$C E$ - Tapping & 74 & 60 & 89 & 81 & 87 & 97 \\
\hline$C E-4 \times 10+$ tapping & 76 & 70 & 95 & 72 & 73 & 92 \\
\hline
\end{tabular}

Cuando se considera la CWT, los resultados se observan en la Tabla 5. Los mejores resultados se obtuvieron con la energía relativa en la población joven (94 \%) con el ejercicio 4x10. Para la población adulta el mejor resultado se obtuvo con la entropía al fusionar el ejercicio del tapping con el 4x10 (85\%).

En algunos casos existe una diferencia marcada entre los resultados obtenidos en la clasificación de pacientes respecto a controles jóvenes y controles adultos, sin embargo, se obtienen tasas de aciertos altas en ambas poblaciones. Es de resaltar que la especificidad es siempre más alta que la sensibilidad, lo que conlleva que el sistema detecta muy bien los sanos, la tasa de falsos positivos se debe mantener baja, lo cual evita malos diagnósticos.

La Tabla 6 muestra el promedio aritmético de la eficiencia del clasificador con las diferentes tareas y características que se han implementado. Puede notarse que, en términos generales, el mejor resultado se obtiene con la CWT en la población joven.

Por otra parte, se ha realizado la clasificación del grupo de jóvenes de acuerdo con las características temporales, con el fin de compararlas con las obtenidas en el dominio tiempo frecuencia. Los resultados se aprecian en la Tabla 7 . La mejor clasificación en el dominio temporal ha sido de $89 \%$ y se ha logrado con el shimmer absoluto y relativo en la tarea $4 \times 10$ y con el jitter absoluto en la tarea del tapping.

\subsection{Evaluación de características}

Las medidas calculadas para el clasificador aportan una gran cantidad de 
información que permite discriminar entre pacientes y jóvenes sanos y entre pacientes y adultos sanos. Para la población joven, los mejores resultados se han obtenido en la tarea del $4 \times 10$ y en la población de adultos con la tarea tapping. La Figura 5 muestra la envolvente de la energía absoluta para el ejercicio del tapping en la población en los espectrogramas y la Fig. 6 para los escalogramas. Las gráficas se muestran solamente para el eje $\mathrm{X}$ de las señales de aceleración, sin embargo, los resultados son muy similares para todos los ejes.

Con respecto a la energía relativa, la Fig. 7 y Fig. 8 muestra la envolvente para el ejercicio del tapping en la misma población. En general el contorno de energía es mayor en los pacientes en las zonas de media y alta frecuencia.

Las Fig. 9 y 10 muestran las envolventes de la entropía espectral por banda de frecuencia. Puede verse que en el análisis del escalograma, las entropías de los controles son mayores en casi todas las bandas, mientras que los escalogramas muestran mayor entropía para los adultos sanos en las bajas frecuencias (alrededor de los $2 \mathrm{~Hz}$ ). Las envolventes de los centroides espectrales se muestran en la Fig. 11 y Fig. 12, donde se aprecian diferencias notables en ambas representaciones con respecto a la información obtenida al considerarse la STFT o la CWT. Esto puede deberse a la diferencia en resolución de la frecuencia de cada una de las transformadas, aunque se generaliza la obtención del $25 \%$ de la energía por debajo de $1 \mathrm{~Hz}, 50 \%$ de la energía alrededor de los $2 \mathrm{~Hz}$ y el $75 \%$ por debajo de los $6 \mathrm{~Hz}$ en ambas representaciones.

Tabla 5. Resultados totales y parciales de la clasificación en el dominio tiempo frecuencia usando la CWT. $\boldsymbol{F C}$ : Fusión de características $\boldsymbol{E}_{\boldsymbol{a}}$ : Energía absoluta. $\boldsymbol{E}_{r}$ : Energía relativa $\boldsymbol{S}$ : Entropía espectral. $\boldsymbol{C} \boldsymbol{E}$ : Centroides espectrales. Fuente: autores

\begin{tabular}{|c|c|c|c|c|c|c|}
\hline \multirow[t]{2}{*}{ Características - tarea } & \multicolumn{3}{|c|}{$\begin{array}{c}\text { Pacientes Vs } \\
\text { Jóvenes }\end{array}$} & \multicolumn{3}{|c|}{$\begin{array}{c}\text { Pacientes Vs } \\
\text { Adultos }\end{array}$} \\
\hline & Acc & Sen & Spe & Acc & Sen & Spe \\
\hline$F C-4 \mathrm{X} 10$ & 94 & 87 & 100 & 68 & 60 & 74 \\
\hline$F C$ - Tapping & 86 & 77 & 95 & 84 & 77 & 90 \\
\hline$F C-4 \times 10+$ tapping & 88 & 83 & 92 & 82 & 77 & 87 \\
\hline$E_{a}-4 \mathrm{X} 10$ & 86 & 80 & 92 & 71 & 67 & 74 \\
\hline$E_{a}-$ Tapping & 79 & 67 & 89 & 82 & 73 & 90 \\
\hline$E_{a}-4 \times 10+$ tapping & 91 & 90 & 92 & 84 & 73 & 92 \\
\hline$E_{r}-4 \mathrm{X} 10$ & 94 & 83 & 92 & 72 & 67 & 79 \\
\hline$E_{r}$-Tapping & 82 & 63 & 95 & 79 & 73 & 85 \\
\hline$E_{r}-4 \times 10+$ tapping & 83 & 83 & 92 & 78 & 73 & 79 \\
\hline$S-4 \mathrm{X} 10$ & 91 & 87 & 95 & 66 & 53 & 77 \\
\hline$S$ - Tapping & 88 & 83 & 92 & 81 & 73 & 87 \\
\hline$S-4 \times 10+$ tapping & 91 & 83 & 97 & 85 & 77 & 92 \\
\hline$C E-4 \mathrm{X} 10$ & 88 & 93 & 95 & 75 & 67 & 77 \\
\hline$C E$ - Tapping & 80 & 70 & 92 & 79 & 67 & 90 \\
\hline$C E-4 \times 10+$ tapping & 88 & 77 & 89 & 76 & 73 & 82 \\
\hline
\end{tabular}

Tabla 6. Resultados promediados de la eficiencia del clasificador ponderado por la cantidad de tareas y conjunto de características. Fuente: autores

\begin{tabular}{ccccc}
\hline & \multicolumn{2}{c}{$\begin{array}{c}\text { Pacientes } \\
\text { Vs Jóvenes }\end{array}$} & \multicolumn{2}{c}{$\begin{array}{c}\text { Pacientes } \\
\text { Vs Adultos }\end{array}$} \\
\cline { 2 - 6 } & CWT & STFT & CWT & STFT \\
Eficiencia & $86.9 \pm$ & $82.8 \pm$ & $77.8 \pm$ & $77.8 \% \pm$ \\
$(\mu \pm \sigma)(\%)$ & $4.6 \%$ & $6.2 \%$ & $5.6 \%$ & $8.7 \%$ \\
\hline
\end{tabular}


Representaciones tiempo-frecuencia basadas en sensores inerciales para caracterizar la marcha en la enfermedad de Parkinson

Tabla 7. Resultados totales y parciales de la clasificación en el dominio espacio-temporal.Shimm abs: shimmer absoluto. Shim rel: shimmer relativo. THD: Distorsión armónica total. TP: Localización en el tiempo de los 3 máximos globales de la señal de posición en el ejercicio del tapping. Fuente: autores

\begin{tabular}{lc}
\hline \multicolumn{1}{c}{ Características - tarea } & $\begin{array}{c}\text { Pacientes Vs. } \\
\text { jóvenes }\end{array}$ \\
\hline Fusión - 4X10 & $\mathbf{8 9 \%}$ \\
Fusión - Tapping & $86 \%$ \\
Fusión - 4x10 + tapping & $79 \%$ \\
Shim. abs.- 4X10 & $89 \%$ \\
Shim. abs.- Tapping & $89 \%$ \\
Shim. abs- 4x10 + tapping & $79 \%$ \\
Shim. rel - 4X10 & $\mathbf{8 9 \%}$ \\
Shim rel.-Tapping & $85 \%$ \\
Shim. rel- 4x10 + tapping & $79 \%$ \\
Jitter abs. tapping & $89 \%$ \\
Jitter rel. tapping & $89 \%$ \\
THD & $81 \%$ \\
TP & $76 \%$ \\
\hline
\end{tabular}

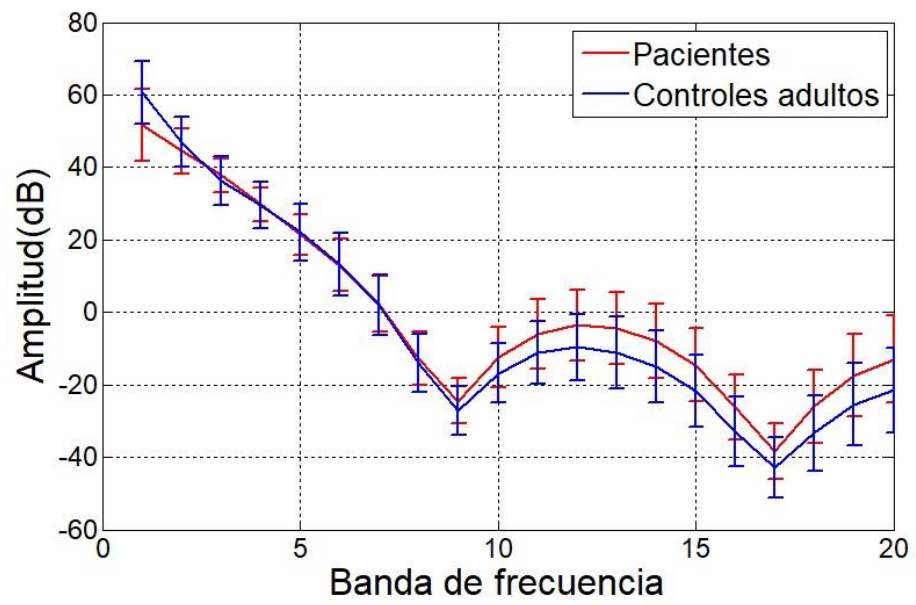

Fig. 5. Envolventes de la energía absoluta obtenida del espectrograma para el ejercicio del tapping para controles adultos mayores y pacientes utilizando el eje X de las señales del acelerómetro. Fuente: autores

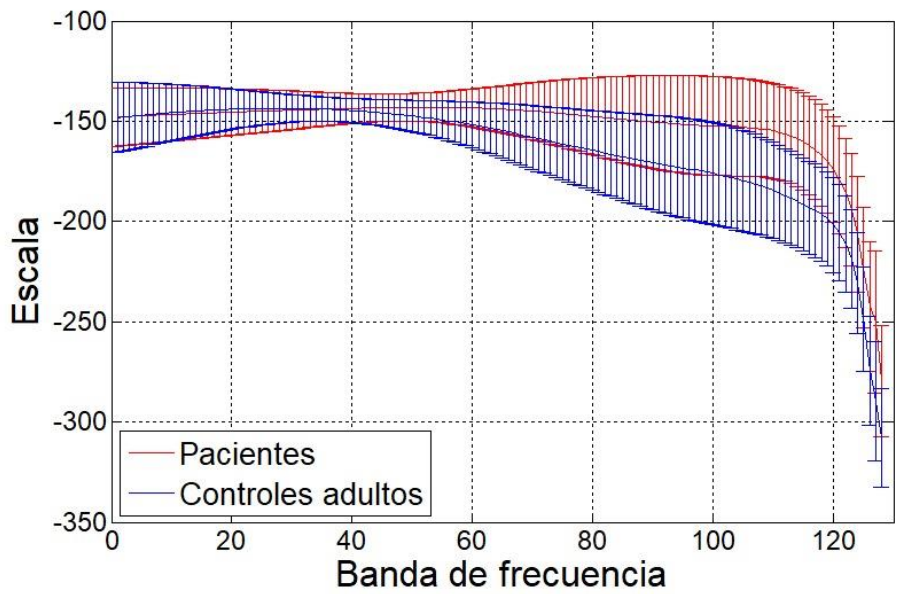

Fig. 6. Envolventes de la energía absoluta obtenida de la CWT para el ejercicio del tapping para controles adultos mayores y pacientes utilizando el eje $\mathrm{X}$ de las señales del acelerómetro. Fuente: autores 
Representaciones tiempo-frecuencia basadas en sensores inerciales para caracterizar la marcha en la enfermedad de Parkinson

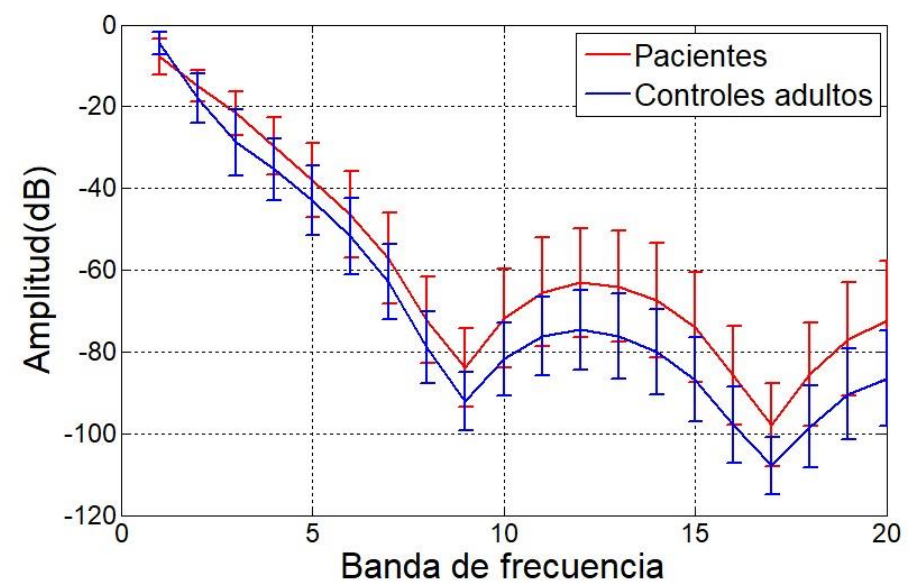

Fig. 7. Envolventes de la energía relativa obtenida del espectrograma para el ejercicio del tapping para controles adultos mayores y pacientes utilizando el eje X de las señales del acelerómetro. Fuente: autores

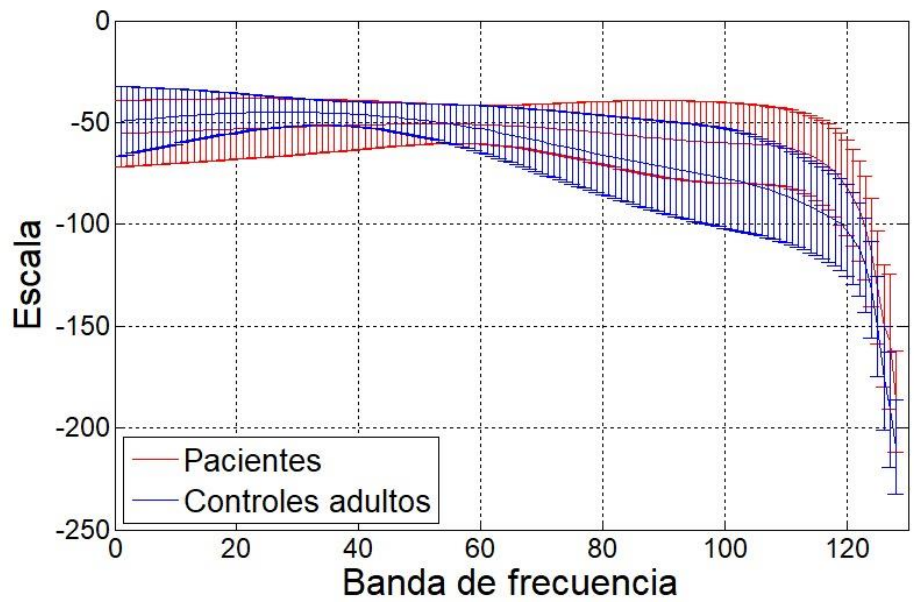

Fig. 8. Envolventes de la energía relativa obtenida de la CWT para el ejercicio del tapping para controles adultos mayores y pacientes utilizando el eje X de las señales del acelerómetro. Fuente: autores

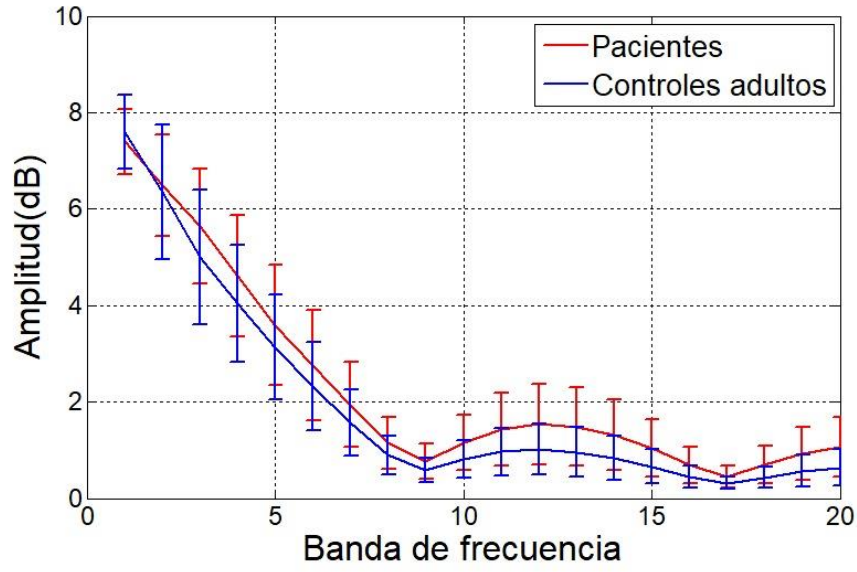

Fig. 9. Envolventes de la entropía espectral obtenida del espectrograma para el ejercicio 4x10 para controles adultos mayores y pacientes utilizando el eje X de las señales del acelerómetro. Fuente: autores 
Representaciones tiempo-frecuencia basadas en sensores inerciales para caracterizar la marcha en la enfermedad de Parkinson

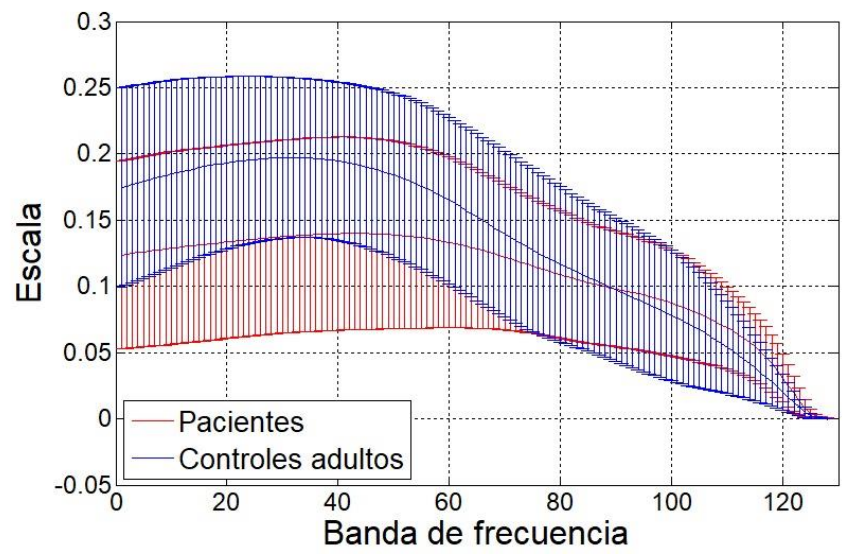

(a)

Fig. 10. Envolventes de la entropía espectral obtenida de la CWT para el ejercicio 4x10 para controles adultos mayores y pacientes utilizando el eje $\mathrm{X}$ de las señales del acelerómetro. Fuente: autores

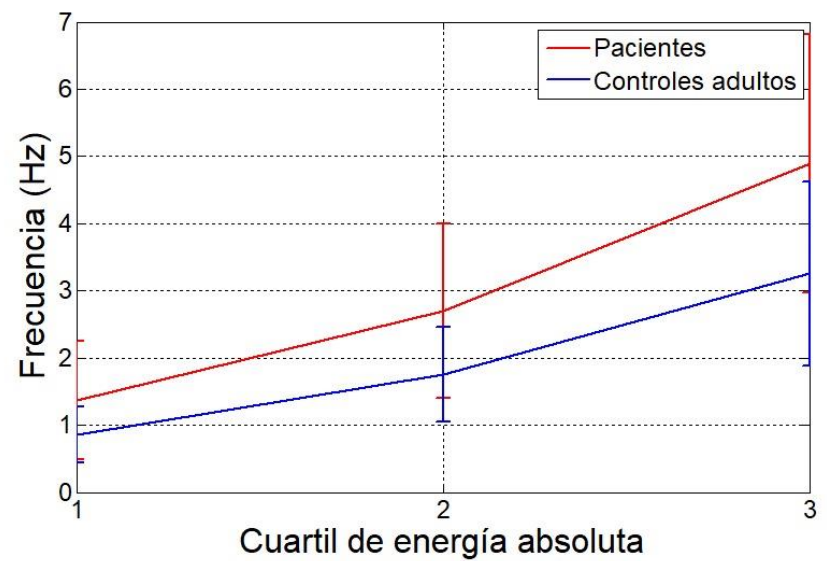

(a)

Fig. 11. Centroides espectrales calculados del espectrograma para el ejercicio del tapping para controles adultos mayores y pacientes utilizando el eje $\mathrm{X}$ de las señales del acelerómetro. Fuente: autores

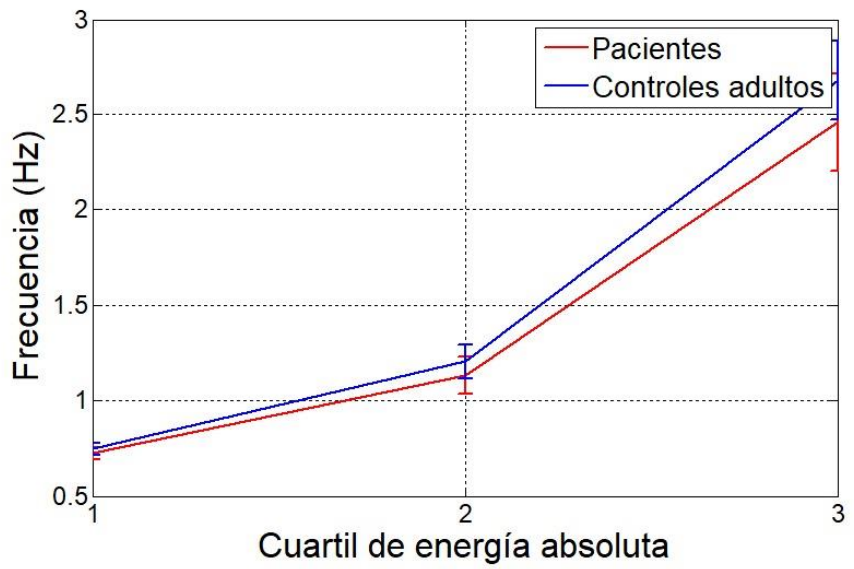

Fig. 12. Centroides espectrales calculados del espectrograma para el ejercicio del tapping para controles adultos mayores y pacientes utilizando el eje $\mathrm{X}$ de las señales del acelerómetro.

Fuente: autores 
Representaciones tiempo-frecuencia basadas en sensores inerciales para caracterizar la marcha en la enfermedad de Parkinson

\section{CONCLUSIONES}

En este trabajo se evalúa el desempeño de medidas basadas en representaciones tiempo frecuencia como la STFT y CWT para clasificar señales de marcha de pacientes con EP y dos grupos de controles sanos con diferentes edades. Sobre cada representación tiempo frecuencia se calculan medidas relacionadas con la energía y la entropía en bandas espectrales. $\mathrm{El}$ primer resultado importante es la diferencia en la eficiencia en casi todas las tareas en la clasificación cuando se considera pacientes respecto a jóvenes o respecto a controles adultos. Esto puede deberse a que en las personas sanas adultas mayores no solamente la EP produce deterioro motriz. El desgaste natural del cuerpo $\mathrm{y}$ otras posibles enfermedades o lesiones pueden tener un efecto negativo en la motricidad.

Las mejores medidas para realizar los diferentes análisis son la energía relativa y la entropía espectral para ambas poblaciones, siendo los centroides espectrales los que menos información aportaron. Otro punto a considerar es que la combinación de tareas y/o características, no necesariamente mejora los resultados de clasificación. De hecho, con todas las características conjuntas, al combinar $4 \mathrm{x} 10$ y tapping, se tiene una disminución del $6 \%$. De la misma manera, el mejor resultado para el ejercicio $4 \times 10$ se obtiene únicamente con la evaluación de la energía relativa.

Los resultados de este trabajo abren el camino para realizar futuras investigaciones en la cual se valore la evolución de las características descritas bajo los efectos de la medicación suministrada para los pacientes. Además, se espera implementar una metodología que permita realizar clasificación multiclase, con la finalidad de clasificar a los pacientes con EP de acuerdo con niveles de la escala MDS-UPDRS y poder con ello predecir su estado neurológico.

\section{AGRADECIMIENTOS}

Este trabajo fue financiado por el CODI de la Universidad de Antioquia por los proyectos PRG 2015-7683 y PR 17-205.

\section{REFERENCIAS}

[1] S. Sveinbjornsdottir, "The clinical symptoms of Parkinson's disease," J. Neurochem., vol. 139, pp. 318-324, Oct. 2016.

[2] A. A. Moustafa et al., "Motor symptoms in Parkinson's disease: A unified framework," Neurosci. Biobehav. Rev., vol. 68, pp. 727740, Sep. 2016.

[3] C. G. Goetz et al., "Movement Disorder Society-sponsored revision of the Unified Parkinson's Disease Rating Scale (MDSUPDRS): Scale presentation and clinimetric testing results," Mov. Disord., vol. 23, no. 15, pp. 2129-2170, Nov. 2008.

[4] R. B. Postuma et al., "MDS clinical diagnostic criteria for Parkinson's disease," Mov. Disord., vol. 30, no. 12, pp. 1591-1601, Oct. 2015.

[5] M. E. Morris, F. Huxham, J. McGinley, K. Dodd, and R. Iansek, "The biomechanics and motor control of gait in Parkinson disease," Clin. Biomech., vol. 16, no. 6, pp. 459-470, Jul. 2001.

[6] J. Barth et al., "Combined analysis of sensor data from hand and gait motor function improves automatic recognition of Parkinson's disease," in International Conference of the Engineering in Medicine and Biology Society (EMBC), 2012, pp. 51225125.

[7] B. Mariani, M. C. Jiménez, F. J. G. Vingerhoets, and K. Aminian, "On-Shoe Wearable Sensors for Gait and Turning Assessment of Patients With Parkinson's Disease," IEEE Trans. Biomed. Eng., vol. 60, no. 1, pp. 155-158, Jan. 2013.

[8] E. Sejdic, K. A. Lowry, J. Bellanca, M. S. Redfern, and J. S. Brach, "A Comprehensive Assessment of Gait Accelerometry Signals in Time, Frequency and Time-Frequency Domains," IEEE Trans. Neural Syst. Rehabil. Eng., vol. 22, no. 3, pp. 603-612, May 2014

[9] J. C. M. Schlachetzki et al., "Wearable sensors objectively measure gait parameters in Parkinson's disease," PLoS One, vol. 12, no. 10, p. e0183989, Oct. 2017.

[10] J. C. Vásquez-Correa et al., "Multi-view representation learning via gcca for multimodal analysis of Parkinson's disease," 
Representaciones tiempo-frecuencia basadas en sensores inerciales para caracterizar la marcha en la enfermedad de Parkinson

in 2017 IEEE International Conference on Acoustics, Speech and Signal Processing (ICASSP), 2017, pp. 2966-2970.

[11] A. Martínez-Ramírez et al., "Frailty assessment based on trunk kinematic parameters during walking," J. Neuroeng. Rehabil., vol. 12, no. 1, p. 48, Dec. 2015.

[12] B. Boashash, "Time-frequency signal analysis and processing: a comprehensive reference," in Time-Frequency Signal Analysis and Processing: A Comprehensive Reference, 2nd ed., Academic Press, 2015, pp. 65-100.

[13] J. R. Orozco-Arroyave et al., "NeuroSpeech: An open-source software for Parkinson's speech analysis," Digit. Signal Process., vol.
77, pp. 207-221, Jun. 2018.

[14] L. Cohen, "Time-frequency distributions-a review," Proc. IEEE, vol. 77, no. 7, pp. 941981, Jul. 1989.

[15] S. Mallat, A wavelet tour of signal processing: the sparse way, 3rd ed. Academic press, 2008.

[16] C. K. Chui, An introduction to wavelets. Academic Press, 2016.

[17] T. Arias-Vergara, J. C. Vásquez-Correa, and J. R. Orozco-Arroyave, "Parkinson's Disease and Aging: Analysis of Their Effect in Phonation and Articulation of Speech," Cognit. Comput., vol. 9, no. 6, pp. 731-748, Dec. 2017. 